\title{
Retreatment with interferon plus ribavirin of chronic hepatitis $C$ non-responders to interferon monotherapy: a meta-analysis of individual patient data
}

\author{
C Cammà, S Bruno, F Schepis, O Lo lacono, P Andreone, A G Gramenzi, A Mangia, \\ A Andriulli, M Puoti, A Spadaro, M Freni, V Di Marco, L Cino, G Saracco, A Chiesa, \\ A Crosignani, N Caporaso, F Morisco, M G Rumi, A Craxì
}

\begin{abstract}
Background and aims: Retreatment with a combination of $\alpha$ interferon (IFN) plus ribavirin of patients with chronic hepatitis $C$ who did not respond to IFN monotherapy has not been assessed in large controlled studies.

Methods: To assess the effectiveness and tolerability of IFN/ribavirin retreatment of non-responders to IFN and to identify predictors of complete (biochemical and virological) sustained response, we performed a meta-analysis of individual data on 581 patients from 10 centres. Retreatment with various IFN schedules (mean total dose 544 mega units) and a fixed ribavirin dose (1000-1200 mg/daily depending on body weight) was given for 24-60 (mean 39.5) weeks.

Results: Biochemical end of treatment and sustained responses were observed in $271 / 581 \quad 146.6 \%$; $95 \%$ confidence interval (Cl) $42.6-50.7 \%$ ) and in $109 / 581(18.7 \% ; 95 \% \mathrm{Cl} 15.6-22.0 \%)$ cases, respectively. Two hundred and six of 532 patients $(38.7 \% ; 95 \% \mathrm{Cl} 34.6-42.9 \%)$ had an end of treatment complete response to retreatment while a complete sustained response occurred in 88 of 559 (15.7\%; $95 \% \mathrm{Cl} 12.8-18.8 \%)$. Fifty four of 581 patients $(9.2 \% ; 95 \% \mathrm{Cl} 7.0-11.7 \%)$ stopped retreatment due to adverse effects. By logistic regression, complete sustained response was predicted independently by age $<45$ years $(p=0.04)$, by normal pretreatment $\gamma$-glutamyltransferase levels $(p=0.01)$, and by a second course total IFN dose of at least 432 mega units $(p=0.008)$.

Conclusions: The overall low probability of effectiveness argues against indiscriminate retreatment of all IFN monotherapy non-responders with IFN/ribavirin. Patients less than 45 years old with normal $\gamma$-glutamyltransferase levels who were retreated with high dose long course combination therapy had a complete sustained response rate of $30 \%$.
\end{abstract}

See end of article for authors' affiliations

Correspondence to: Dr C Cammà, Via Alcide De Gasperi 50, 90100 Palermo, Italy:

camma@ismeda.pa.cnr.it

Accepted for publication 2 April 2002
S ince the early 1990s, at least $80 \%$ of patients with chronic hepatitis C, treated with standard $\alpha$ interferon (IFN) monotherapy ( 3 mega units (MU) thrice weekly for 24 weeks), have not responded to this therapy. ${ }^{1}$ A large cohort of IFN monotherapy non-responders (that is, subjects who did not reach an end of treatment response) still exists within the pool of subjects with chronic hepatitis C. Furthermore, the natural history of the disease seems to be worse in these patients than in responders to IFN. ${ }^{2}$ In fact, there is a higher rate of end stage liver disease development as well as hepatocellular carcinoma in non-responders. Therefore, an effective regimen of retreatment is a major goal in their long term management.

In 1998, four large international multicentre randomised controlled trials (RCTs) showed that IFN and ribavirin combination therapy was significantly more effective than IFN alone both in untreated and in relapsed patients. ${ }^{3-6}$ However, the effectiveness of combination therapy for non-responders to IFN monotherapy is less clear. In 1999, the European Association for the Study of the Liver (EASL) Consensus Statement did not recommend IFN and ribavirin combination therapy, or any other treatment, for subjects with chronic hepatitis $C$ who had previously not responded to IFN monotherapy. ${ }^{7}$ Two recent meta-analyses of nine and 12 RCTs, ${ }^{8} 9$ comparing combination therapy to retreatment with IFN monotherapy in non-responders, concluded that IFN and ribavirin are more effective than IFN alone, although the overall benefit was small with only a $7 \%$ of pooled risk difference for sustained virological response and with a pooled withdrawal rate due to intolerance of treatment of $9 \%$ for the combination. ${ }^{9}$ This ben- efit is of questionable clinical relevance, making it unfeasible to indiscriminately retreat all non-responders with such a low likelihood of response.

Important questions still remain unanswered. Is combination retreatment for non-responders cost effective? Are there differences in the effectiveness of IFN retreatment between cirrhotic and non-cirrhotic patients, between different HCV genotypes, and between different second course regimens? Due to the use of summary data, both previous meta-analyses failed to identify the most responsive subgroups. The aims of this meta-analysis of individual patient data (MIPD) were: (1) to critically reassess the efficacy and tolerability of IFN and ribavirin retreatment in obtaining a sustained response in non-responders, (2) to identify the best retreatment schedule to be used, and finally ( 3 ) to identify possible predictors of a complete (biochemical and virological) sustained response to retreatment.

\section{MATERIALS AND METHODS}

This multicentre study was planned to pool patient data from a large number of individuals from European tertiary referral

Abbreviations: $\mathrm{HCV}$, hepatitis $C$ virus; IFN, $\alpha$ interferon; MIPD, meta-analysis of individual patient data; ETR, end of treatment response; $S R$, sustained response; RCT, randomised controlled trial; $M U$, mega units; ALT, alanine transaminase; ROC, receiver operating characteristic; $A \cup C$, area under the curve; OR, odds ratio; NNT, number needed to be retreated. 
liver units in an attempt to define the efficacy and tolerability of IFN and ribavirin retreatment in non-responders. Potential participating centres were identified by screening MEDLINE (January 1998-December 2000), including only English articles, with the following medical subject headings: hepatitis C, IFN, and ribavirin. The computer search was supplemented with manual searches of reference lists for all available review articles, primary studies, abstracts from meetings, and bibliographies of books. To avoid publication bias, both published and unpublished studies were included. Therefore, all trialists who took part in the meta-analysis were asked to help in identifying studies. Studies were included in the meta-analysis if they were randomised or non-randomised controlled trials or prospective cohort studies fulfilling the following criteria: (1) included non-responders, defined according to the EASL International Consensus Conference on Hepatitis $C^{7}$ who had received a first course of IFN at a dose of at least $3 \mathrm{MU}$ thrice weekly for 12 weeks (partial responders, breakthrough patients, and relapsers were excluded); (2) started retreatment within 24 months after the end of the first course; (3) retreated all patients between January 1996 and January 2000; and (4) used a retreatment IFN dose of at least $9 \mathrm{MU} /$ week and a ribavirin dose of at least $800 \mathrm{mg} /$ day. Nine centres in Italy and one centre in Spain, all with reported experience in this field, met the inclusion criteria and agreed to make data available. Among the included studies, three were RCTs, ${ }^{1014}{ }^{16}$ one was a non-RCT, ${ }^{17}$ and six were prospective cohort studies. ${ }^{11-13} 15$ Five studies, which accounted for 312 patients (54\%), were reported as full papers ${ }^{10-14}$ while three of the studies, which accounted for 189 patients $(32 \%)$, were abstracts. ${ }^{15-17}$ Data from the remaining two cohort studies, which included 80 patients (14\%), are still unpublished.

After checking for inconsistencies and possible duplications, the final study database consisted of 581 consecutive non-responders who met the following criteria for inclusion before the first treatment: over 18 years of age; alanine transaminase (ALT) level greater than 1.5 times the upper normal limit for at least six months before entry; anti-HCV positivity by Enzyme Immune Assay, and HCV-RNA positivity by any method before entry; histological features of chronic hepatitis (with or without cirrhosis); serum hepatitis B surface antigen negative; human immunodeficiency virus antibody negative; antinuclear antibody negative; self reported complete abstinence from alcohol; and no more than one course of IFN monotherapy.

\section{Virology}

HCV antibodies were tested on pretreatment samples by second or third generation Enzyme Immune Assay (Ortho Diagnostics, Raritan, New Jersey, USA). HCV-RNA was detected in serum either by b-DNA version 2.0 (assay detection limit 160000 genomes $/ \mathrm{ml}$ ) or by polymerase chain reactions performed using onsite materials or those obtained commercially (Amplicor; Roche, Basel, Switzerland; detection limit $1 \times 10^{3}$ genomes $/ \mathrm{ml}$ ). Results were expressed qualitatively. Quantification was performed by b-DNA version 2.0 in 409 cases $(70.3 \%)$. Genotyping was performed by reverse hybridisation line probe assay. ${ }^{18}$

\section{Definition of response}

Responses to the first treatment with IFN and to retreatment with IFN/ribavirin were defined as ALT normalisation (biochemical response), HCV-RNA negative (virological response), or both (complete response), and on the basis of the timing of the results as either an end of treatment response (ETR) or a sustained response (SR), as defined by the EASL Consensus Conference on Hepatitis C. ${ }^{19}$ ALT levels were measured monthly during the treatment period and posttreatment follow up. Evaluation of therapeutic efficacy was performed by an "intention to treat" strategy.
Table 1 Baseline characteristics of the patients

\begin{tabular}{|c|c|c|}
\hline $\mathrm{n}$ & 581 & \\
\hline Men (No (\%)) & 386 & (66.4) \\
\hline Mean age $(y)$ & 45.9 & $(10.8)$ \\
\hline Mean PLT $\left(10^{3} / \mathrm{mm}^{3}\right)$ & 172.5 & $(54.6)$ \\
\hline Mean $\gamma$-glutamyltransferase (ULN) & 1.68 & $(1.48)$ \\
\hline Mean ALT (ULN) & 3.17 & $(2.19)$ \\
\hline \multicolumn{3}{|l|}{ HCV genotype (No (\%)) } \\
\hline 1 & 311 & $(53.5)$ \\
\hline 2 & 150 & $(25.8)$ \\
\hline 3 & 55 & (9.4) \\
\hline 4 & 28 & (4.8) \\
\hline Mixed or other & 37 & (6.3) \\
\hline \multicolumn{3}{|l|}{ Serum HCV-RNA $\dagger$} \\
\hline No of copies/ml & $2 \times 10^{6}$ & $\left(6.9 \times 10^{6}\right)$ \\
\hline$\geqslant 2 \times 10^{6}$ copies $/ \mathrm{ml}(\mathrm{No}(\%))$ & 68 & $(11.7)$ \\
\hline \multicolumn{3}{|l|}{ Liver biopsy (No (\%)) } \\
\hline \multicolumn{3}{|l|}{ Chronic hepatitis (Metavir F1 to F3) } \\
\hline Metavir A1-A2 & 250 & (43) \\
\hline Metavir A3-A4 & 267 & (45.9) \\
\hline Cirrhosis (Metavir F4) & 64 & (11) \\
\hline \multicolumn{3}{|l|}{ First IFN course (No (\%)) } \\
\hline Standard dose $(234 \mathrm{MU}) \ddagger$ & 383 & (65.9) \\
\hline Medium dose (>234-468 MU) & 130 & (22.3) \\
\hline High dose (>468 MU) & 68 & $(11.7)$ \\
\hline Mean total dose (MU) & 295.8 & (236.4) \\
\hline \multicolumn{3}{|l|}{ Second IFN course (No (\%)) } \\
\hline \multicolumn{3}{|l|}{ Therapeutic regimens (total dose): } \\
\hline $3 \mathrm{MU}$ tiw for 6 months (234 MU) & 84 & (14.4) \\
\hline $5 \mathrm{MU}$ tiw for 6 months (390 MU) & 55 & $(9.4)^{*}$ \\
\hline $6 \mathrm{MU}$ tiw for 6 months (468 MU) & 86 & (14.8) \\
\hline $3 \mathrm{MU}$ tiw for 12 months (468 $\mathrm{MU}$ ) & 135 & $(23.2)$ \\
\hline $5 \mathrm{MU}$ tiw for 12 months $(780 \mathrm{MU}$ ) & 137 & (23.5) \\
\hline $6 \mathrm{MU}$ tiw for 12 months (936 MU) & 84 & (14.4) \\
\hline Mean total dose (MU) & 544.3 & $(265.1)$ \\
\hline \multicolumn{3}{|l|}{ Length of retreatment (No $(\%)$ ) } \\
\hline 6 months & & \\
\hline 12 months & 356 & (61.3) \\
\hline \multicolumn{3}{|l|}{ Type of IFN for retreatment } \\
\hline Recombinant $\alpha 2 b$ & 529 & (91.1) \\
\hline Leucocytic $\alpha \mathrm{N}-3$ & 33 & (5.7) \\
\hline
\end{tabular}

Values are mean (SD) or No (\%).

*21 patients received a course of $5 \mathrm{MU}$ of IFN daily.

†Assessed in 409 patients.

$\ddagger 3$ mega units three times weekly for six months.

PLT, platelets; ULN, upper limit of normal; ALT, alanine transaminase $\mathrm{HCV}$, hepatitis $C$ virus; IFN, $\alpha$ interferon; MU, mega units; tiw, three times weekly.

\section{Statistical analysis}

Univariate data comparisons among groups were performed using $\chi^{2}$ tests for proportions and Student's $t$ tests for means. On the basis of experience gathered from naive patients and from preliminary evidence on retreated patients, ${ }^{20}$ we selected as candidate predictors of biochemical and virological SR, age, sex, platelet counts, ALT and $\gamma$-glutamyltransferase levels, baseline liver histology, HCV genotype, HCV-RNA levels before retreatment, and amount of IFN received during the first and second cycles. All analyses used a two sided $\mathrm{p}=0.05$ significance level and were conducted with the PROC LOGISTIC subroutine in SAS (SAS Institute, Inc., Cary, North Carolina, USA). ${ }^{21}$

Variables significant on multivariate analysis were used to generate a prediction rule. For each case a score was calculated and a probability of response assigned giving the set of values for the variables. The main statistical methods for the logit function and logistic regression have been described elsewhere. ${ }^{22}$ From the scoring of all predictors significant at multivariate analysis, a simple incremental rule (from 1 to 8 ) was established to evaluate each individual patient in the test and training sets. The $\beta$ coefficient of this score was then tested for significance in the test set.

The sensitivity and specificity of each rule to the response status were estimated by means of a receiver operating characteristic (ROC) curve, determined by the Hanley and 
Table 2 Results of retreatment according to baseline features and regimens administered

\begin{tabular}{|c|c|c|c|c|c|c|c|c|}
\hline \multirow[b]{3}{*}{ All patients } & \multicolumn{4}{|c|}{ End of treatment response } & \multicolumn{4}{|c|}{ Sustained response } \\
\hline & \multicolumn{2}{|c|}{ Biochemical (\% (No)) } & \multicolumn{2}{|c|}{ Complete $(\%(\mathrm{No}))$} & \multicolumn{2}{|c|}{ Biochemical (\% (No)) } & \multicolumn{2}{|c|}{ Complete (\% (No) } \\
\hline & 46.6 & $(271 / 581)$ & 38.7 & $(206 / 532)$ & 18.7 & $(109 / 581)$ & 15.7 & (88/559) \\
\hline \multicolumn{9}{|l|}{ Cirrhosis } \\
\hline Present & 29.6 & $(19 / 64)$ & 27.5 & $(16 / 58)$ & 14 & $(9 / 64)$ & 12.5 & $(8 / 64)$ \\
\hline Absent & 48.7 & $(252 / 517)$ & 40 & $(180 / 474)$ & 19.3 & $(100 / 517)$ & 16.1 & $(80 / 495)$ \\
\hline$p$ Value & \multicolumn{2}{|c|}{0.004} & \multicolumn{2}{|c|}{0.065} & \multicolumn{2}{|l|}{0.30} & \multicolumn{2}{|l|}{0.44} \\
\hline \multicolumn{9}{|l|}{ Genotype } \\
\hline 1 & 45.6 & $(142 / 311)$ & 30.4 & $(92 / 302)$ & 15.1 & $(47 / 311)$ & 14.4 & $(43 / 297)$ \\
\hline Non-1 & 47.7 & (129/270) & 49.5 & (114/230) & 22.9 & $(62 / 270)$ & 17.1 & $(45 / 262)$ \\
\hline $\mathrm{p}$ Value & \multicolumn{2}{|l|}{0.60} & \multicolumn{2}{|c|}{0.001} & \multicolumn{2}{|c|}{0.016} & \multicolumn{2}{|l|}{0.38} \\
\hline \multicolumn{9}{|l|}{ HCV-RNA } \\
\hline$\geqslant 2 \times 10^{6}$ copies $/ \mathrm{ml}$ & 30.8 & $(21 / 68)$ & 30 & $(18 / 60)$ & 22 & $(15 / 68)$ & 17.6 & $(12 / 68)$ \\
\hline$<2 \times 10^{6}$ copies $/ \mathrm{ml}$ & 49.5 & (169/341) & 37.3 & (115/308) & 18.1 & $(62 / 341)$ & 16.3 & $(55 / 336)$ \\
\hline $\mathrm{p}$ Value & \multicolumn{2}{|c|}{0.005} & \multicolumn{2}{|c|}{0.27} & \multicolumn{2}{|c|}{0.45} & \multicolumn{2}{|c|}{0.79} \\
\hline \multicolumn{9}{|l|}{ IFN retreatment regimens } \\
\hline Standard dose (234 MU) & 39.2 & $(33 / 84)$ & 31.6 & $(25 / 79)$ & 8.3 & $(7 / 84)$ & 6.1 & $(5 / 82)$ \\
\hline Medium dose (>234-468 MU) & 46.3 & $(133 / 287)$ & 34 & $(91 / 267)$ & 15.3 & $(44 / 287)$ & 13.2 & $(36 / 272)$ \\
\hline High dose $(>468 \mathrm{MU})$ & 50 & $(105 / 210)$ & 48.3 & $(90 / 186)$ & 27.6 & $(58 / 210)$ & 22.9 & $(47 / 205)$ \\
\hline $\mathrm{p}$ Value & \multicolumn{2}{|l|}{0.24} & \multicolumn{2}{|c|}{0.003} & \multicolumn{2}{|c|}{0.001} & \multicolumn{2}{|c|}{0.001} \\
\hline \multicolumn{9}{|l|}{ Length of retreatment } \\
\hline 6 months & 44 & $(99 / 225)$ & 41.2 & $(83 / 201)$ & 11.1 & $(25 / 225)$ & 10.1 & (21/208) \\
\hline 12 months & 48.3 & $(172 / 356)$ & 58.3 & (193/331) & 23.6 & $(84 / 356)$ & 19 & $(67 / 351)$ \\
\hline $\mathrm{p}$ Value & 0.31 & & 0.001 & & 0.001 & & 0.005 & \\
\hline 1 st $\rightarrow 2$ nd IFN course & & & & & & & & \\
\hline Standard $\rightarrow$ standard & 38.4 & $(20 / 52)$ & 39.5 & $(19 / 48)$ & 13.4 & $(7 / 52)$ & 9.6 & $(5 / 52)$ \\
\hline Standard $\rightarrow$ medium/high & 49.5 & $(164 / 331)$ & 39.5 & $(121 / 306)$ & 21.7 & $(72 / 331)$ & 17.2 & $(55 / 319)$ \\
\hline Medium/high $\rightarrow$ standard & 40.6 & $(13 / 32)$ & 19.3 & $(6 / 31)$ & 0 & $(0 / 32)$ & 0 & $(0 / 30)$ \\
\hline Medium/high $\rightarrow$ medium/high & 44.5 & $(74 / 166)$ & 40.8 & $(60 / 147)$ & 18 & $(30 / 166)$ & 17.7 & $(28 / 158)$ \\
\hline $\mathrm{p}$ Value & 0.35 & & 0.15 & & 0.016 & & 0.044 & \\
\hline Type of IFN for retreatment & & & & & & & & \\
\hline Recombinant $\alpha 2 b$ & 46.7 & $(256 / 548)$ & 52.3 & $(261 / 499)$ & 18.8 & $(103 / 548)$ & 15.5 & $(82 / 526)$ \\
\hline Leucocytic $\alpha \mathrm{N}-3$ & 45.4 & $(15 / 33)$ & 45.4 & $(15 / 33)$ & 18.1 & $(6 / 33)$ & 18.1 & $(6 / 33)$ \\
\hline$p$ Value & 0.88 & & 0.44 & & 0.93 & & 0.69 & \\
\hline
\end{tabular}

McNeil method..$^{23}$ Each curve shows the capacity of the related model to discriminate between those who respond and those who fail to respond to IFN at different cut off values. The larger the area under the curve (AUC), the better the discriminating ability of each rule (range 0.5 (chance performance) to 1.0 (perfect prediction)).

We performed a cross validation study by dividing the data randomly into two portions, one for model development (training set) and the other for model validation (test set). The discriminatory ability of the models was assessed in the training and test sets by plotting ROC curves and comparing AUC values. $^{24}$

\section{RESULTS}

\section{Patient characteristics}

Baseline characteristics of the 581 patients included are shown in table 1 . The proportion of cases with cirrhosis $(11 \%)$ was relatively low. A total of $383(65.9 \%)$ patients had received a standard first IFN course ( 3 MU thrice weekly for 24 weeks): $22.3 \%$ were treated with a total IFN dose of 234-468 MU while in 68 patients $(11.7 \%)$ a total dose higher than $468 \mathrm{MU}$ had been given.

The retreatment regimens of the studies are shown in table 1. Large variability in retreatment schedules between centres was found for the total dose of IFN (234-936 MU), duration of retreatment (24-52 weeks), and the single dose of IFN ( 3 and $6 \mathrm{MU}$ ). Ribavirin was administered orally twice a day at a total daily dose of $1000 \mathrm{mg}$ for patients weighing $75 \mathrm{~kg}$ or less and at $1200 \mathrm{mg}$ for those $>75 \mathrm{~kg}$.

\section{Outcome}

ALT levels, as shown in table 2, were normal by the end of treatment in 271 of 581 subjects $(46.6 \%$; $95 \%$ confidence interval (CI) 42.6-50.7\%). A complete ETR occurred in 206 of
532 patients (38.7\%; 95\% CI 34.6-42.9\%) in whom HCV-RNA was tested. It is noteworthy that the best rates of complete ETR were achieved in patients retreated with a high dose IFN course $(48.3 \%, 95 \%$ CI $44.2-52.3 \%)$ and in those who did not have HCV genotype 1 (49.5\%, 95\% CI 45.4-53.5\%).

One hundred and nine of 581 patients had a biochemical SR to retreatment (18.7\%; 95\% CI 15.6-22.0\%). A complete SR was achieved in 88 of 559 patients ( $15.7 \% ; 95 \%$ CI $12.8-18.8 \%$ ) in whom serum HCV-RNA was tested six months after the end of retreatment. We failed to demonstrate any significant difference in the probability of complete SR between different HCV genotypes, between different baseline liver histologies, and between different pre-retreatment HCV-RNA levels (table 2 ). A complete SR (table 3 ) was achieved in 47 of 205 subjects (22.9\%; 95\% CI $17.4-28.9 \%)$ retreated with a high dose IFN combination regimen. In contrast, low rates of complete SR were obtained in patients receiving standard retreatment regimens, both when previously treated with a standard $(9.6 \%)$ or high dose $(0 \%)$ first IFN course. We did not find any significant difference in the probability of biochemical $(p=0.93)$ or complete SR $(p=0.69)$ according to the type of IFN (recombinant or leucocytic) administered in the second course.

\section{Safety}

Retreatment with IFN and ribavirin was not universally tolerated. Side effects leading to withdrawal from both IFN and ribavirin retreatment occurred in 54 of 581 patients $(9.2 \%$; 95\% CI $7.0-11.7 \%)$. Mean time of treatment discontinuation was 11.9 (8.7) weeks. No life threatening events were observed during treatment.

Mean decrease in haemoglobin from baseline was 2.6 ( 1.78$)$ $\mathrm{g} / \mathrm{dl}$. In 48 of 581 subjects $(8.2 \%)$ dyspnoea, probably triggered by low haemoglobinaemia, was reported. Anaemia was managed by reducing ribavirin to $600 \mathrm{mg} /$ day in 78 of 581 patients 
Table 3 Univariate analysis of predictors associated with biochemical and virological sustained responders to interferon (IFN) and ribavirin retreatment

\begin{tabular}{|c|c|c|c|c|c|}
\hline \multirow[b]{2}{*}{ Men (No $(\%))$} & \multicolumn{2}{|c|}{$\begin{array}{l}\text { Complete sustained } \\
\text { responders }(\mathrm{n}=88)\end{array}$} & \multicolumn{2}{|c|}{$\begin{array}{l}\text { All others } \\
(\mathrm{n}=471)\end{array}$} & \multirow{2}{*}{$\begin{array}{l}\mathrm{p} \text { Value } \\
0.44\end{array}$} \\
\hline & \multicolumn{2}{|c|}{$55 \quad(62.5)$} & \multicolumn{2}{|c|}{$314 \quad(66.6)$} & \\
\hline Mean age (y) & \multirow{2}{*}{\multicolumn{2}{|c|}{$43.5(12.1)$}} & \multicolumn{2}{|c|}{$46.5(10.6)$} & 0.019 \\
\hline Mean PLT $\left(10^{3} / \mathrm{mm}^{3}\right)$ & & & \multicolumn{2}{|c|}{$173.4(55.2)$} & 0.85 \\
\hline Mean ALT (ULN) & \multirow{2}{*}{\multicolumn{2}{|c|}{$\begin{array}{l}3.0(2.1) \\
1.2(1.3)\end{array}$}} & \multirow{2}{*}{\multicolumn{2}{|c|}{$\begin{array}{l}3.1(2.1) \\
1.7(1.5)\end{array}$}} & 0.68 \\
\hline Mean $\gamma$-glutamyltransferase (ULN) & & & & & 0.01 \\
\hline \multicolumn{5}{|l|}{ HCV genotype (No (\%)) } & \multirow[t]{3}{*}{0.38} \\
\hline Type 1 & 43 & (48.8) & 254 & (53.9) & \\
\hline Other than type 1 & 45 & (51.1) & 217 & $(46.0)$ & \\
\hline \multicolumn{5}{|l|}{ Serum HCV-RNA (No (\%))* } & \multirow[t]{3}{*}{0.79} \\
\hline$<2 \times 10^{6}$ copies $/ \mathrm{ml}$ & 55 & (82) & 281 & (83.3) & \\
\hline$\geqslant 2 \times 10^{6}$ copies $/ \mathrm{ml}$ & 12 & (18) & 56 & (16.6) & \\
\hline \multicolumn{5}{|l|}{ Liver biopsy (No (\%)) } & \multirow{5}{*}{0.061} \\
\hline \multicolumn{5}{|l|}{ Chronic hepatitis (Metavir F1 to F3) } & \\
\hline Metavir A1-A2 & 31 & (35.2) & 217 & (46) & \\
\hline Metavir A3-A4 & 49 & (55.6) & 198 & (42) & \\
\hline Cirrhosis (Metavir F4) & 8 & (9.0) & 56 & $(11.8)$ & \\
\hline \multicolumn{5}{|l|}{ Second IFN course (No (\%)) } & \multirow[t]{3}{*}{0.0037} \\
\hline Total dose $<432 \mathrm{MU}$ & 14 & (15.9) & 149 & $(31.6)$ & \\
\hline Total dose $\geqslant 432 \mathrm{MU}$ & 74 & (84.1) & 322 & (68.3) & \\
\hline \multirow{2}{*}{\multicolumn{5}{|c|}{$\begin{array}{l}\text { 1st } \rightarrow \text { 2nd IFN course } \\
\text { Standard } \rightarrow \text { medium/high (No (\%)) }\end{array}$}} & \multirow[t]{4}{*}{0.26} \\
\hline & & & & & \\
\hline No & 33 & (37.5) & 207 & (43.9) & \\
\hline Yes & 55 & (62.5) & 264 & (56) & \\
\hline \multicolumn{6}{|c|}{$\begin{array}{l}\text { Values are mean (SD) or No (\%). } \\
\text { *Assessed in } 409 \text { patients. } \\
\text { PLT, platelets; ULN, upper limit of normal; ALT, alanine transaminase; HCV, hepatitis } C \text { virus; IFN, } \\
\alpha \text { interferon; MU, mega units. }\end{array}$} \\
\hline
\end{tabular}

(13.4\%; $95 \%$ CI $10.7-16.2 \%)$ or by its discontinuation in 27 of 581 subjects $(4.6 \% ; 95 \%$ CI $3.0-6.4 \%)$. Haemoglobin levels returned to baseline within eight weeks after the end of treatment, and no patient required transfusions. The following additional adverse events were also observed: pruritus, rash, nausea, depression, insomnia, anorexia, and weight loss.

Variables associated with complete sustained response Univariate and multivariate analyses were performed to identify predictors of complete SR after retreatment. Univariate comparison of variables between complete SR and all other patients is reported in table 3. Young age, normal pretreatment $\gamma$-glutamyltransferase levels, and retreatment with a total IFN dose $\geqslant 432$ MU were significantly associated with a complete SR to retreatment. Absence of cirrhosis at baseline liver biopsy was of marginal significance $(p=0.061)$. An increased dose strategy (that is, a medium/high total IFN dose for retreatment of patients who had received a standard IFN dose during the first course) was used in 319 of 581 subjects (54.9\%; $95 \%$ CI 50.9-59.0\%). This strategy did not significantly increase the rate of success: the proportion of complete SR was 55/319 $(17.2 \%$; $95 \%$ CI $13.2-21.5 \%)$ in subjects in whom the dose was increased and 33/240 (13.7\%; 95\% CI 9.5-18.3\%) in patients who received the same dose of IFN $(p=0.26)$.

We performed a subgroup analysis in patient retreated with a total IFN dose $\geqslant 432$ MU to evaluate whether there was a different rate of complete SR according to duration of treatment. As shown in table 3, a complete SR was achieved in 74 of 396 subjects $(18.6 \%, 95 \%$ CI $14.9-22.6 \%)$ retreated with a high dose regimen. Among them, the likelihood of a complete SR was significantly lower when the high dose was delivered over a period of $\leqslant 26$ weeks $(7 / 73$ (9.5\%); $95 \%$ CI $3.5-19.5 \%)$ versus over a period of $>26$ weeks $(67 / 323$ (20.7\%); 95\% CI 16.4-25.3\%) ( $\mathrm{p}=0.027)$.

Multivariate analysis (table 4) showed that, in decreasing order of significance, the following were independent predictors of complete SR: retreatment with a total IFN dose $\geqslant 432$ MU (odds ratio (OR) 2.25), normal pretreatment $\gamma$-glutamyltransferase levels (OR 0.54 ), and age $<45$ years (OR 0.62 ). These variables were then used to construct a model encompassing all patients grouped into classes of risk according to the presence of one, two, or three of these factors (fig 1A). As expected, the likelihood of complete SR gradually decreased from the "best" to the "worst" class. Only three of 55 patients $(5.4 \%)$ in the worst class (age $\geqslant 45$ years, elevated pretreatment $\gamma$-glutamyltransferase levels, and retreatment with a total IFN dose $<432 \mathrm{MU}$ ) had a complete SR. At the other extreme, a subject in the best class (age $<45$ years, normal pretreatment $\gamma$-glutamyltransferase levels, and retreatment with a total IFN dose $\geqslant 432 \mathrm{MU}$ ) had a likelihood of a complete SR of $30.5 \%$ (36 of 118 actual cases).

Table 4 Logistic regression model to predict complete sustained response in non-responders retreated with interferon and ribavirin

\begin{tabular}{|c|c|c|c|c|c|c|}
\hline Variable & Code & $\beta$ & SE & $\mathrm{p}$ Value & Odds ratio & $95 \% \mathrm{Cl}$ \\
\hline Age & $\begin{array}{l}0:<45 \\
1: \geqslant 45\end{array}$ & -0.47 & 0.23 & 0.04 & 0.62 & $0.38-0.98$ \\
\hline$\gamma$-glutamyltransferase & $\begin{array}{l}\text { 0: Normal } \\
\text { 1: Elevated }\end{array}$ & -0.61 & 0.26 & 0.01 & 0.54 & $0.32-0.90$ \\
\hline $\begin{array}{l}\text { Second IFN course total dose } \\
\text { (MU) }\end{array}$ & $\begin{array}{l}0:<432 \mathrm{MU} \\
1: \geqslant 432 \mathrm{MU}\end{array}$ & 0.81 & 0.31 & 0.008 & 2.25 & $1.22-4.14$ \\
\hline
\end{tabular}

Model $\chi^{2}=17.8$ with $3 \mathrm{df}, \mathrm{p}<0.001$

IFN, $\alpha$ interferon; $M U$, mega units. 

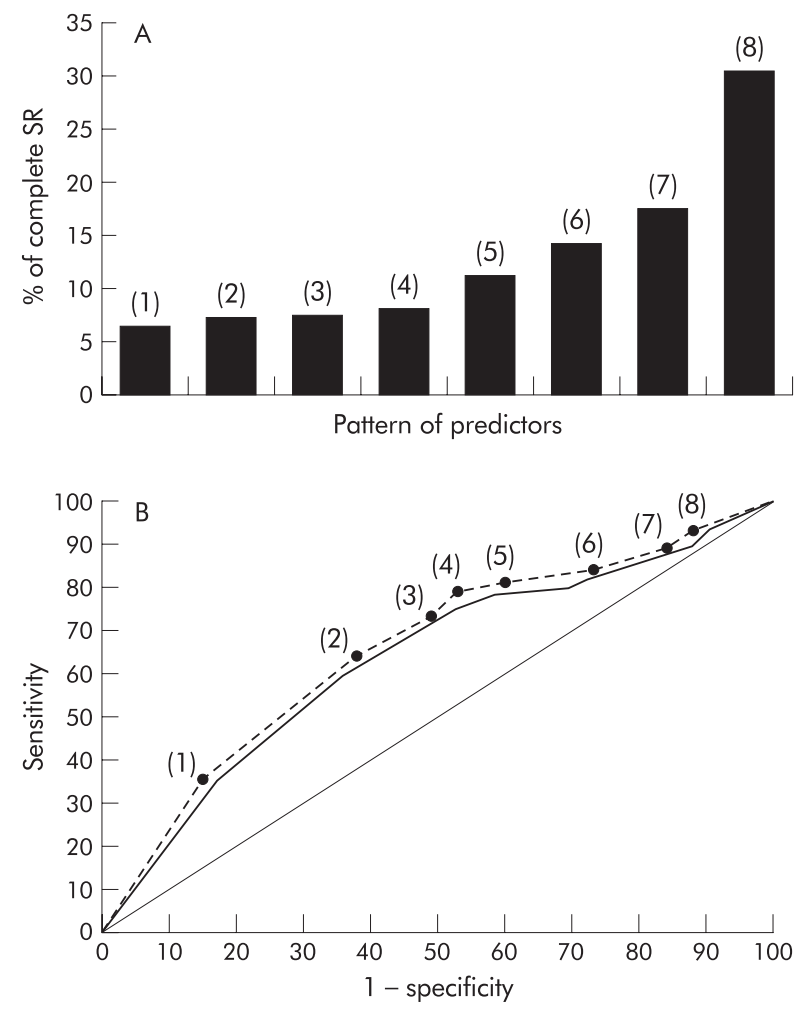

Figure 1 Complete sustained response rate according to specific patterns of predictors $(A)$ and receiver operating characteristics curves $(B)$ for the rule predicting complete sustained response in the training set (broken line) and in the test set (solid line). The diagonal line indicates no discriminating power (training set: area under the curve (AUC) 0.69, SEM 0.046; test set: AUC 0.67; SEM 0.048). In $(A)$ and $(B)$, numbers in parentheses indicates the following pattern of predictors: $1=$ age $\geqslant 45$ years, elevated pretreatment $\gamma$-glutamyltransferase levels, and second interferon (IFN) course total dose $<432$ mega units (MU); $2=$ age $\geqslant 45$ years, normal pretreatment $\gamma$-glutamyltransferase levels, and second IFN course total dose $<432 \mathrm{MU} ; 3=$ age $\geqslant 45$ years, elevated pretreatment $\gamma$-glutamyltransferase levels, and second IFN course total dose $\geqslant 432$ MU; $4=$ age $<45$ years, elevated pretreatment $\gamma$-glutamyltransferase levels, and second IFN course total dose <432 MU; 5=age <45 years, normal pretreatment $\gamma$-glutamyltransferase levels, and second IFN course total dose $<432 \mathrm{MU} ; 6=$ age $<45$ years, elevated pretreatment $\gamma$-glutamyltransferase levels, and second IFN course total dose $\geqslant 432 \mathrm{MU} ; 7=$ age $\geqslant 45$ years, normal pretreatment $\gamma$-glutamyltransferase levels, and second IFN course total IFN dose $\geqslant 432 \mathrm{MU} ; 8=$ age $<45$ years, normal pretreatment $\gamma$-glutamyltransferase levels, and second IFN course total dose $\geqslant 432$ MU.

\section{Cross validation}

To assess the validity of the model, we randomly derived a test set of 290 patients from the entire data set. Figure 1B shows the ROC curves for the rules predicting a complete SR in the training set (281 patients: AUC 0.69, SEM 0.046) and in the test set (278 patients: AUC 0.67, SEM 0.048). Areas were not statistically different (z 0.19; two sided p value 0.84 ). As an example, at the cut off value of 4 (age $<45$ years, elevated pretreatment $\gamma$-glutamyltransferase levels, and retreatment with a total IFN dose $<432 \mathrm{MU}$ ) the model correctly identified $79 \%$ of complete SR patients at a cost of inappropriately predicting $53 \%$ of non-SR patients.

\section{DISCUSSION}

This MIPD, combining data from 10 centres that in total retreated almost 600 non-responders to IFN monotherapy, shows that retreatment with a combination of IFN and ribavirin achieves a complete SR in $15 \%$ of cases at a cost of a $10 \%$ withdrawal due to drug intolerance. The reported value for efficacy is in keeping with the results of two recently published conventional meta-analyses, ${ }^{89}$ and does not lend support to indiscriminate retreatment of IFN non-responders. Due to its low overall efficacy and tolerability, as well as the ensuing poor cost effectiveness, the decision to retreat non-responders with IFN and ribavirin still remains difficult. ${ }^{25}$ The key issue is identification of patients with a higher likelihood of responding to retreatment with the combination therapy. Although the response to treatment was less than $20 \%$, even in the most responsive subgroups, both previously published meta-analyses ${ }^{89}$ failed in identifying any differences in baseline patient characteristics significantly associated with a complete SR.

Drawing firm conclusions based on the results of the two previously reported meta-analyses is hampered by the considerable heterogeneity in the trials included in these two conventional meta-analyses performed on aggregated data. These summary results describe only between study, and not between patient, variation because they reflect group averages rather than individual data. The pooled estimate does not adequately describe the findings of a meta-analysis when the observed effects in individual studies differ substantially. ${ }^{26}$ Therefore, when a significant heterogeneity in baseline risk is found, more detailed treatment comparisons can be achieved only by a MIPD. ${ }^{27}$

Our analysis showed that age $<45$ years, normal preretreatment $\gamma$-glutamyltransferase level, and a second IFN course dose $\geqslant 432 \mathrm{MU}$ independently predicted a complete SR. In the subgroup of patients with these most favourable predictors, the probability of a complete SR was approximately $30 \%$ and the number of patients needed to be retreated (NNT) to obtain one complete SR was 3.3, while coexistence of the most unfavourable predictors raised the NNT to 15.8 . Modelling the indication for retreatment of non-responders with combination therapy on these predictors would maximize its cost effectiveness. An internal cross validation assessment of the reliability of this multivariate model substantiates this statement. Although Cheng and colleagues ${ }^{8}$ suggested that the benefit of combination therapy may be higher (14.7\% virological SR) in patients infected with HCV genotypes other than 1, we could not confirm significant improvement in complete SR according to genotype. The two other predictors of unresponsiveness to retreatment-that is, more advanced age and raised $\boldsymbol{\gamma}$-glutamyltransferase levels-are well known predictors of insensitivity to IFN therapy. ${ }^{20} 2829$ Thus it is not surprising to find that they are strong predictors of unresponsivess to IFN and ribavirin in a group of patients in whom other factors such as cirrhosis, genotype, and viral load have been eliminated by a priori selection for unresponsiveness to the previous IFN course.

Many studies have tried to identify the ideal dose of combination therapy that would maximise the cost effectiveness of retreatment in the individual patient. As the results of published studies are inconclusive or conflicting, the last Consensus Development Conference on Hepatitis C did not recommend any regimen of retreatment for non-responders. A recent large multicentre $\mathrm{RCT}^{30}$ has shown that retreatment with a regimen of $5 \mathrm{MU}$ three times weekly for 12 months produced a significantly better virological SR than low dose short duration therapy (3 MU thrice weekly for six months). In contrast, Di Bisceglie et al failed to demonstrate a significant difference in the response rate between 24 and 48 weeks of IFN and ribavirin retreatment. ${ }^{31}$ Our MIPD provides evidence that retreatment with a high dose (at least $432 \mathrm{MU}$ ) long course ( $>26$ weeks) of IFN is the best option for a complete SR. We did not confirm the observation of Cummings and colleagues, ${ }^{9}$ suggesting a difference in the likelihood of complete SR between recombinant and human leucocytic IFN. However, the number of patients treated with leucocytic IFN in our meta-analysis was small, and minor differences could have been lost. 
Recently, a small RCT of IFN, ribavirin, and amantadine for retreatment of non-responders ${ }^{32}$ reported an impressive advantage of triple antiviral therapy in terms of virological SR ( $48 \%$ in the triple therapy group versus $5 \%$ in the IFN and ribavirin group), although at the cost of significantly more intolerance to treatment. Although the ages of the cases in the two treatment groups were comparable, no information on other predictors was available; in particular, the study did not report the rates of SR to retreatment according to dose, duration, and number of previous courses. As the dose administered during the first course is a key factor in the selection of non-responders and could influence the likelihood of response to combination therapy, further large scale multicentre RCTs which are in progress will prove useful to substantiate the benefit of retreatment of non-responders with triple therapy.

As with all meta-analyses, this study has the potential limitation of the generalisability of results to new populations and settings. Meta-analyses are likely to have poor external validity when included studies all use the same limited patient population or are all conducted in a single setting. As non-responders are a heterogeneous population, we decided to include studies with different designs, conducted in different settings, and including non-responders to different first IFN courses retreated with different regimens. We are confident that this approach increases the generalisability of our results. In contrast, our MIPD and both the previously published meta-analyses by Cummings and colleagues ${ }^{9}$ and Cheng and colleagues ${ }^{8}$ included mostly Italian studies, limiting the broad application of the results.

Concerning retreatment of non-responders to IFN monotherapy, we believe the available evidence is sufficient to conclude that: (1) the overall low probability of clinical benefit and the relevant costs of therapy and adverse reactions to drugs argue against indiscriminate use of IFN/ribavirin for all non-responders; (2) patients less than 45 years of age and with normal pretreatment $\gamma$-glutamyltransferase levels can be retreated with combination therapy, with a 30\% likelihood of response; and (3) retreatment must be done with high dose IFN for 48 weeks, regardless of the dose and timing received in the first course.

\section{Authors' affiliations}

C Cammà, Istituto Metodologie Diagnostiche Avanzate, Consiglio Nazionale delle Ricerche, and Cattedra e Unità Operativa di Gastroenterologia, University of Palermo, Palermo, Italy

S Bruno, A Chiesa, A Crosignani, Istituto di Scienze Biomediche, San Paolo University of Milano, Italy

F Schepis, Dipartimento di Medicina Sperimentale e Clinica "G

Salvatore", University of Catanzaro, Italy

O Lo lacono, V Di Marco, L Cino, A Craxì, Cattedra e Unità

Operativa di Gastroenterologia, University of Palermo, Italy

P Andreone, A G Gramenzi, Dipartimento di Medicina Interna,

Epatologia, University of Bologna, Italy

A Mangia, A Andriulli, Divisione di Gastroenterologia, Casa Sollievo della Sofferenza, S Giovanni Rotondo, Italy

M Puoti, Clinica Malattie Infettive e Tropicali, University of Brescia, Italy

A Spadaro, M Freni, Clinica Medica la, University of Messina, Italy

G Saracco, Cattedra e Unità Operativa di Gastroenterologia, Ospedale Molinette, University of Torino, Italy

N Caporaso, F Morisco, Dipartimento di Scienze Alimentari, University Federico II of Napoli, Italy

M G Rumi, Cattedra e Unità Operativa di Gastroenterologia, IRCCS

Ospedale Maggiore, University of Milano, Italy

\section{REFERENCES}

1 Cammà $C$, Giunta $M$, Pinzello GB, et al. Chronic hepatitis $C$ and interferon alpha: conventional and cumulative meta-analyses of randomized controlled trials. Am J Gastroenterol 1999;94:581-95.

2 Cammà $\mathrm{C}$, Di Marco V, Lo lacono $\mathrm{O}$, et al. Long-term course of interferon-treated chronic hepatitis C. J Hepatol 1998:28:531-7.

3 Poynard T, Marcellin P, Lee SS, et al. Randomised trial of interferon $\alpha-2 b$ plus ribavirin for 48 weeks or for 24 weeks versus interferon $\alpha-2 b$ plus placebo for 48 weeks for treatment of chronic infection with hepatitis C virus. Lancet 1998;352:1426-32.
4 McHutchison JG, Gordon SC, Schiff ER, et al. Interferon alfa-2b alone or in combination with ribavirin as initial treatment for chronic hepatitis C. N Engl J Med 1998;339:1485-92.

5 Davis GL, Esteban-Mur R, Rustgi VK, et al. Interferon alfa-2b alone or in combination with ribavirin for the treatment of relapse of chronic hepatitis C. N Engl J Med 1998;339:1493-9.

6 Reichard O, Norkrans G, Fryden A, et al. Randomised, double-blind, placebo-controlled trial of interferon $\alpha-2 b$ with and without ribavirin for chronic hepatitis C. Lancet 1998;351:83-7.

7 EASL International Consensus Conference on Hepatitis C. Paris, 26-28, February 1999, Consensus Statement. European Association for the Study of the Liver. J Hepatol 1999;30:956-61.

8 Cheng SJ, Bonis PA, Lau J, et al. Interferon and ribavirin for patients with chronic hepatitis $C$ who did not respond to previous interferon therapy: a meta-analysis of controlled and uncontrolled trials. Hepatology $2001 ; 33: 231-40$.

9 Cummings KJ, Lee SM, West ES, et al. Interferon and ribavirin vs interferon alone in the re-treatment of chronic hepatitis $C$ previously nonresponsive to interferon. JAMA 2001;285:193-9.

10 Sostegni R, Ghisetti V, Pittaluga F, et al. Sequential versus concomitant admistration of ribavirin and interferon alfa-n 3 in patients with chronic hepatitis $C$ non responding to interferon alone: results of a randomized, controlled study. Hepatology 1998;28:341-6.

11 Lo lacono O, Castro A, Diago M, et al. Interferon alfa-2b plus ribavirin for chronic hepatitis $C$ patients who have not responded to interferon monotherapy. Aliment Pharmacol Ther 2000;14:463-9.

12 Spadaro A, Freni MA, Ajello A, et al. Interferon retreatment of patients with chronic hepatitis C. A long-term follow-up. Hepatogastroenterology $1999 ; 46: 3229-33$

13 Di Marco V, Vaccaro A, Ferraro D, et al. High-dose prolonged combination therapy in non-responders to interferon monotherapy for chronic hepatitis C. Aliment Pharmacol Ther 2001;15:953-8.

14 Puoti M, Cadeo GP, Putzolu V, et al. Pilot dose-finding trial on interferon alpha in combination with ribavirin for the treatment of chronic hepatitis $\mathrm{C}$ in patients not responding to interferon alone. Dig Liver Dis 2001;33:163-72.

15 Morisco F, Canestrini C, Astretto S, et al. Therapeutic efficacy of reinforced vs. standard combination therapy schedule (IFN alfa $2 b+$ ribavirin) in chronic hepatitis $\mathrm{C}$ patients not responding or relapsing to IFN alone. (abstract). Hepatology 1999;30:198A

16 Andreone P, Cursaro C, Gramenzi A, et al. High dose of interferon $\alpha$ (IFN) plus ribavirin (RIBA) for 6 or 12 months in non responder (NR) patients with chronic hepatitis $\mathrm{C}(\mathrm{CHC})$ : results of a randomized trial. $J$ Hepatol 2000;32(suppl 2): 115

17 Fargion S, Bruno S, Borzio $M$, et al. Combination of interferon alpha and ribavirin in chronic hepatitis $C$ non responders to interferon monotherapy: role of genotype and age. J Hepatol 2001;34(suppl 1):205.

18 Stuyver L, Rossau R, Wyseur A, et al. Typing of hepatitis $C$ virus isolates and characterization of new subtypes using a line probe assay. J Gen Virol 1993;74: 1093-203.

19 Craxì A, Cammà C, Giunta M. Definition of response to antiviral therapy in chronic hepatitis C. J Hepatol 1999;31(suppl 1):160-7.

20 Cammà $C$, Giunta $M$, Chemello L, et al. Chronic hepatitis $C$ : interferon retreatment of relapsers. A meta-analysis of individual patient data. Hepatology 1999:30:801-7.

21 SAS Technical Report. SAS/STAT Software. Changes and enhancements, release 6.07. Cary, NC: SAS Institute Inc, 1992

22 Wasson JH, Sox HC, Neff RK, et al. Clinical prediction rules: applications and methodological standards. N Engl J Med 1985;313:793-9.

23 Hanley JA, McNeil BJ. The meaning and use of the area under a receiver operating characteristic (ROC) curve. Radiology 1982;143:29-36.

24 Hanley JA, McNeil BJ. A method of comparing the areas under receiver operating characteristic curve derived from the same cases. Radiology 1983;148:839-48.

25 Koff RS. Nonresponse to interferon in chronic hepatitis C. Re-treatment redux. JAMA 2001;285:212-14

26 DerSimonian R, Levine RJ. Resolving discrepancies between a meta-analysis and a subsequent large controlled trial. JAMA 1999;282:664-70.

27 Trikalinos TA, loannidis JP. Predictive modeling and heterogeneity of baseline risk in meta-analysis of individual patient data. J Clin Epidemiol $2001 ; 54: 245-52$

28 Poynard T, McHutchison J, Goodman Z, et al. Is an "à la carte" combination interferon alfa- $2 b$ plus ribavirin regimen possible for the first line treatment in patients with chronic hepatitis C? Hepatology 2000;31:211-18

29 Heathcote J. Antiviral therapy of patients with chronic hepatitis C. Semin Liver Dis 2000;20:185-99.

30 Saracco G, Ciancio A, Olivero A, et al. A randomised 4-arm multicentre study of interferon alfa- $2 \mathrm{~b}$ plus ribavirin in the treatment of patients with chronic hepatitis $C$ not responding to interferon alone. Hepatology 2001;34:133-8.

31 Di Bisceglie AM, Thompson J, Smith-Wilkaitis N, et al. Combination of interferon and ribavirin in chronic hepatitis $\mathrm{C}$ : re-treatment of nonresponders to interferon. Hepatology 2001;33:704-7.

32 Brillanti S, Levatesi F, Masi L, et al. Triple antiviral therapy as a new option for patients with interferon nonresponsive chronic hepatitis $C$. Hepatology 2000;32:630-4. 